\title{
Once BCG Unresponsive, Always BCG Unresponsive: An Open Letter to the FDA to Enhance Recruitment into Clinical Trials in Bladder Cancer
}

Ashish M. Kamat ${ }^{\mathrm{a}, *}$, Seth Lerner ${ }^{\mathrm{b}}$, Peter Black ${ }^{\mathrm{c}}$, Joaquim Bellmunt ${ }^{\mathrm{d}}$, Colin Dinney ${ }^{\mathrm{a}}$, Noah M. Hahn ${ }^{\mathrm{e}}$, Michael O'Donnell ${ }^{\mathrm{f}}$ and Diane Z. Quale ${ }^{\mathrm{g}}$

${ }^{a}$ Department of Urology, The University of Texas, MD Anderson Cancer Center, Houston, TX, USA

${ }^{\mathrm{b}}$ Department of Urologic Oncology, Baylor College of Medicine, TCGA Bladder Cancer, Houston, TX, USA

${ }^{\mathrm{c}}$ University of British Columbia, Vancouver, BC, Canada

${ }^{\mathrm{d}}$ Department of Medicine, Harvard Medical School, Bladder Cancer Center, Dana-Farber Cancer Institute, Boston, MA, USA

${ }^{\mathrm{e}}$ Department of Oncology and Urology, Johns Hopkins University School of Medicine, Baltimore, MD, USA

${ }_{\mathrm{f}}^{\mathrm{f}}$ Departments of Urology and Urologic Oncology, The University of Iowa, Iowa City, Iowa, IA, USA

${ }^{\mathrm{g}}$ BCAN (Bladder Cancer Advocacy Network), Baltimore, MD, USA

The FDA is to be commended on drafting a guidance document [1] to assist sponsors in the clinical development of drugs for treatment of patients with NMIBC, especially those who have BCGunresponsive disease. Herein, we are writing about a specific issue that has come to light in the last few months, reflecting real world experiences with clinical trial patients on single arm studies with BCG Unresponsive NMIBC.

\section{BACKGROUND}

In their guidance document the FDA defines BCG-unresponsive disease as: "Persistent high-grade disease or recurrence within 6 months of receiving at least two courses of intravesical BCG (at least

*Correspondence to: Ashish M. Kamat, Department of Urology, The University of Texas, MD Anderson Cancer Center, Houston, TX, USA. E-mail: akamat@mdanderson.org.
5 of 6 induction doses and at least 2 of 3 maintenance doses); or T1 high-grade disease at the first evaluation following induction BCG alone (at least 5 of 6 induction doses)" [1-3]. The FDA has recognized that "Patients with BCG-unresponsive disease are extremely unlikely to benefit from further therapy with BCG and represent a unique population for study of new therapies." and that "Single-arm trials are appropriate in clinical settings where a randomized, controlled trial is either unethical or not feasible". Using this guidance, clinical trials have been developed - and several are ongoing - where patients who fit the BCG Unresponsive criteria and are either not fit for or refuse, a radical cystectomy, are able to enroll on a clinical trial of intervention ' $\mathrm{X}$ '.

\section{SPECIFIC SITUATION}

In the conduct of these trials, we have encountered a situation that is best depicted with an illustrative 
example: Patient $\mathrm{Z}$ is diagnosed with CIS of the bladder in March 2016. She elects to receive BCG and undergoes induction with 6 weekly treatments, then received 3 weeks of maintenance therapy. On September 15, 2016, she is evaluated and diagnosed with BCG Unresponsive CIS. Patient $\mathrm{Z}$ refuses to undergo radical cystectomy, and by virtue of meeting inclusion criteria, is able to enroll on a clinical trial of agent X. Three months later - in January of 2017, she undergoes surveillance and is found to have persistent CIS. At this point, she comes off the clinical trial for agent $\mathrm{X}$. She is again counseled on the recommendation for radical cystectomy which she adamantly refuses. There are several other clinical trials available for BCG Unresponsive NMIBC but at this point she is no longer eligible for a second clinical trial as she is out of window for the strict inclusion criteria. At this point, the only option she has is to choose treatment with valrubicin (with its known low efficacy) or non-approved salvage intravesical therapy.

\section{SOLUTION}

As a group, it is our request that a patient such as this, who meets the definition of BCG Unresponsive NMIBC and is treated with one or more rounds of bladder cancer directed therapy but does not achieve a disease free state, be considered eligible for subsequent clinical trials in the BCG Unresponsive setting.
In other words, once a patient meets the criteria to be BCG Unresponsive, they should be able to participate in sequential trials until they experience a response. This should of course, as with all such trials, be under the strict supervision of an expert physician and with due diligence to appropriate re-staging so as to minimize any risk to the patient. This would allow us to provide patients with potentially curable options, with the recognition that while radical cystectomy is still the preferred option, patients who enrolled in such trials do so by virtue of being medically unfit for, or staunchly against, radical cystectomy in the first place. Such patients should not be denied effective therapy.

\section{REFERENCES}

[1] FDA Draft Guidance. BCG-Unresponsive Nonmuscle Invasive Bladder Cancer: Developing Drugs and Biologics for Treatment Guidance for Industry. Last accessed June 5, 2017. https://www.fda.gov/downloads/Drugs/GuidanceComplianc eRegulatoryInformation/Guidances/UCM529600.pdf

[2] Lerner SP, Dinney C, Kamat A, Bivalacqua TJ, Nielsen M, O'Donnell M, Schoenberg MP, Steinberg G. Clarification of bladder cancer disease states following treatment of patients with intravesical BCG. Bladder Cancer 2015;1(1):29-30.

[3] Kamat AM, Sylvester RJ, Böhle A, Palou J, Lamm DL, Brausi M, Soloway M, Persad R, Buckley R, Colombel M, Witjes JA. Definitions, end points, and clinical trial designs for non-muscle-invasive bladder cancer: Recommendations from the International Bladder Cancer Group. J Clin Oncol 2016;34(16):1935-44. 